\title{
Screening for urinary tract colonisation prior to corticosteroid administration in acute multiple sclerosis relapses: validation of an updated algorithm
}

Fergus O'Herlihy, MB BCh BAO ${ }^{\mathrm{a}}$, Nevin A John, MBBS, FRACP ${ }^{\mathrm{b}}$, Vivien Li, MBBS, FRACP ${ }^{\text {,e }}$, Bernadette Porter, MSc c , Lucy Lyons, BSNmc ${ }^{c}$, Martin Rakusa, DM, PhD ${ }^{\mathrm{d}}$, Carmel Curtis, PhD, MRCP, FRCPath ${ }^{\mathrm{c}}$, Jalesh Panicker, $\mathrm{MD}, \mathrm{PhD}^{\mathrm{e}}$, Jeremy Chataway, $\mathrm{MA}, \mathrm{PhD}^{\mathrm{b}, \mathrm{c}}$

${ }^{a}$ University College Dublin School of Medicine and Medical Science, Dublin, Ireland

${ }^{\mathrm{b}}$ Queen Square MS Centre, Department of Neuroinflammation, UCL Institute of Neurology, London, UK

${ }^{c}$ National Hospital for Neurology and Neurosurgery, Queen Square, London, UK

${ }^{\mathrm{d}}$ Department of Neurology, University Medical Centre Maribor, Slovenia

e Department of Uro-Neurology, National Hospital for Neurology and Neurosurgery and UCL Institute of Neurology, Queen Square, London, UK

Address for correspondence:

Professor Jeremy Chataway, Box 112, National Hospital for Neurology and Neurosurgery, Queen Square, WC1N 3BG, London, UK

Email: j.chataway@ucl.ac.uk; Fax: 02076799485; Telephone: 02034483018

Funding: This research did not receive any specific grant from funding agencies in the public, commercial, or not-for-profit sectors.

Disclosures: JC has received support from the Efficacy and Mechanism Evaluation Programme and Health Technology Assessment Programme (NIHR); UK Multiple Sclerosis Society and National Multiple Sclerosis Society; National Institute for Health Research University College London Hospitals (NIHR-UCLH) Biomedical Research Centre (BRC) and University College London. In the last three years, he has been a local principal investigator for trials in multiple sclerosis funded by: Receptos, Novartis and Biogen Idec, and has received an investigator grant from Novartis outside this work. He has taken part in Advisory Boards/consultancy for Roche, Merck, MedDay, Biogen and Celgene. JNP is supported in part by funding from the United Kingdom's Department of Health NIHR Biomedical Research Centres funding scheme.

Abbreviations: High-dose corticosteroids (HDC), intravenous (IV), multiple sclerosis (MS), urinary tract infections (UTIs), leucocyte esterase (LE), negative predictive value (NPV), positive predictive value (PPV), mid-stream urine (MSU), standard deviation (SD), confidence interval (CI), people with multiple sclerosis (PwMS) 


\begin{abstract}
Introduction: To evaluate an updated algorithm in the detection of urinary tract infection (UTI) prior to high-dose corticosteroid treatment in acute relapses in multiple sclerosis (MS). This updated algorithm aimed to decrease the unnecessary use of antibiotics, whilst maintaining accuracy and safety.

Methods: Prospective cohort study of 471 consecutive patients with MS relapses in a hospital-based outpatient acute relapse clinic. 172 patients met exclusion criteria, leaving 299 patients for analysis. Patients underwent urine dipstick and were treated for UTI if 2 or more of: nitrites, leukocyte esterase and cloudy urine were positive. Patients with confirmed acute MS relapse were treated with high dose intravenous or oral methylprednisolone.
\end{abstract}

Results: Significant bacteriuria $\left(>10^{5}\right.$ colonies $\left./ \mathrm{mL}\right)$ was present in $33(11 \%, 95 \%$ CI $8-15)$ patients. The algorithm sensitivity and specificity was $24 \%$ and $94 \%$ respectively; the negative predictive value was $91 \%$. The overall accuracy of the algorithm was $87 \%$. No adverse sequelae were identified in 25 patients who received high dose methylprednisolone in the presence of an untreated UTI.

Conclusion: With an improved specificity, this updated algorithm addresses previous issues concerning the unnecessary prescription of antibiotics, whilst improving accuracy and maintaining safety.

Abstract word count: 188

Key words: Urinary tract colonisation, acute MS relapse treatment, multiple sclerosis, urine dipstick 


\section{Introduction}

The role of high-dose corticosteroids (HDC) in treating significant clinical relapses of multiple sclerosis (MS) is well established, with a variety of intravenous (IV) and oral regimes[1]. Ideally, patients suffering such a relapse would be given HDC as soon as the diagnosis becomes apparent. However, such is the prevalence of bladder dysfunction in MS (roughly $80 \%$ ), that it is common practice to screen all patients with possible relapses for the presence of bacteriuria before steroid administration[2,3]. Urinary tract infections (UTIs) have been linked to worsening of neurological symptoms, and implicated as triggers of acute MS relapses[4-6]. In the context of an acute worsening of neurologic function, it is advised to treat those with bacteriuria with appropriate antimicrobials[7]. Furthermore, untreated UTIs can develop into severe infections, and there have been reports of pyelonephritis, urosepsis and death following high-dose methylprednisolone administration[8].

Ruling out UTI prior to HDC treatment is both critically important and clinically challenging. While urinary culture from a mid-stream urine sample has long been the gold-standard for identifying infection, results generally take at least 48 hours. Withholding steroids while awaiting culture results is not desirable, but identifying UTIs on a symptomatic basis alone is generally not possible, as MS patients frequently experience dysuria, urinary frequency and incontinence, or have indwelling urinary catheters at baseline[9].

Urine microscopy is often used in the inpatient setting to provide preliminary results pending culture report. This approach, however, is not suitable for the outpatient MS relapse clinic for a number of reasons. As highlighted by others, microscopy is time-consuming and expensive, and microscopic pyuria is an unreliable marker of infection in this population[10,11]. Many studies have shown this approach to be equivalent or inferior to urinary dipstick[12,13].

Our group previously reported on an accurate, safe and efficient screening tool for identifying UTIs in the outpatient MS relapse clinic[14]. Using this algorithm (version 1), a urinary dipstick was performed on all patients with a relapse. Those with either positive urinary nitrites or leucocyte esterase (LE) were deemed to have a high likelihood of a UTI, and received empirical antibiotics along with HDC. This algorithm allowed all patients to be treated with HDC for their relapse if clinically indicated. It yielded a specificity of $78 \%$ and sensitivity of $65 \%$; and a negative predictive value (NPV) of $96 \%$ for UTI. $11 \%$ of patients had a proven UTI. One issue with the algorithm, however, was a high false positive rate, with $18 \%$ of patients treated with antibiotics unnecessarily. Concerns over unnecessary prescribing of antibiotics, including the risks of adverse reactions and development of antimicrobial resistance, led to us attempting to refine this algorithm.

Previous studies have shown that assessing urinary appearance can be helpful in detecting UTIs in patient populations similar to ours. Indeed, of all typical signs and symptoms of UTIs, the presence of "cloudy urine" has been shown to be the most accurate indicator of infection in these patients, with a sensitivity of $66 \%$ and an overall accuracy of $83 \%[15]$. As a consequence of this data, we decided to include "cloudy urine" in our updated algorithm (version 2) (Figure 1). We aimed to reduce the false positive rate of the previous algorithm while maintaining accuracy, safety and efficiency. We now describe the performance of this second version with a new cohort of patients suffering from a significant relapse. 


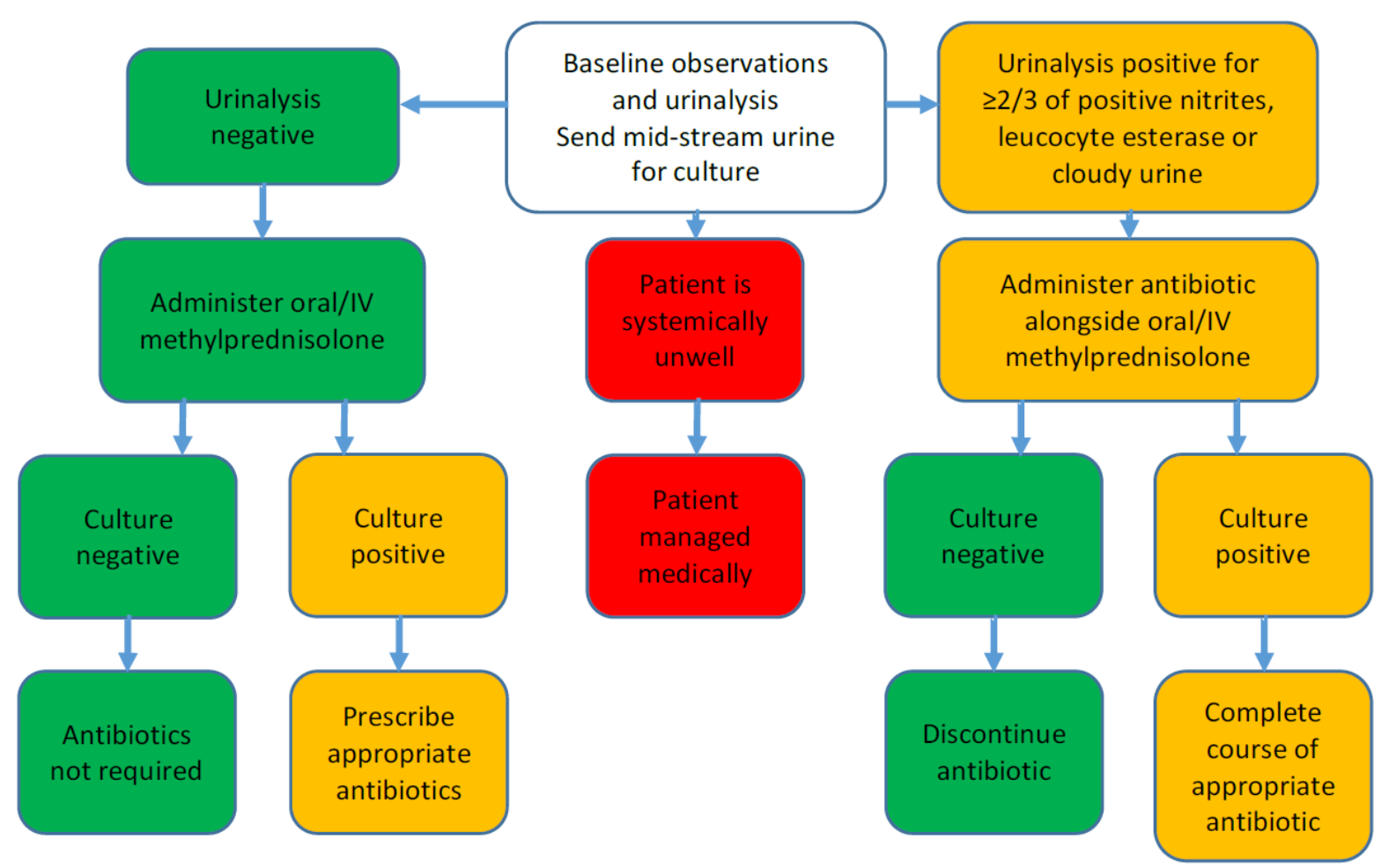

Figure 1: Updated algorithm (version 2) for screening UTIs prior to administering HDC for acute MS relapse.

\section{Methods}

\subsection{Test participants and exclusion criteria}

Data on 471 sequential patients who attended the outpatient MS relapse clinic at the National Hospital for Neurology and Neurosurgery, Queen Square, London between June 2014 and January 2017 was recorded. The clinical process has been previously described[14], and the major inclusion criteria were that patients had to be suffering an acute/subacute MS relapse of moderate severity, necessitating treatment with HDC. The main exclusion criteria were the use of urinary catheters (both indwelling catheters and those performing intermittent self-catheterisation) and the presence of signs of systemic infection, classified as fever, rigors or renal angle tenderness.

\subsection{Test methods}

For urinary dipstick testing we used Bayer Multistix 10SG Reagent Strips (Bayer Corp., Diagnostics Division, Elkhart, IN, USA). The reported dipstick sensitivity for leucocytes was 10-25 WBC/microliter, and 13-22 microM for nitrites. Urine was classified as cloudy or not based on direct visual inspection by nursing staff. Our amended algorithm (version 2) is outlined in Figure 1. Patients with two or more of positive nitrites, LE or cloudy urine were deemed to have a 'positive urine result' and were therefore treated for a UTI. All other patients were deemed to have a negative urine result and therefore not immediately diagnosed as having a UTI. Patients with a positive urine result were treated with empirical antibiotics until formal culture was available. These, as per hospital policy, were nitrofurantoin modified release $100 \mathrm{mg}$ oral taken once daily or if nitrofurantoin was contraindicated, oral trimethoprim 200mg taken twice daily. Antibiotics were prescribed in conjunction with methylprednisolone.

Mid-stream urine (MSU) samples were sent for all patients, and were the gold standard reference for our algorithm. One-microliter samples were plated on quarter CHROMagar BBL (BD Diagnostic Systems, Sparks, MD, USA) plates, incubated at $37^{\circ} \mathrm{C}$ and examined 15-24 hours after plating. A positive culture was defined as pure growth of at least 100 colony forming units of a pathogenic organism (equivalent to $10^{5}$ colonies per $\mathrm{ml}$ ). Mixed growth was not considered pathological. Any organisms not recognisable on CHROMagar were identified by VITEK® two bioMerieux, with antibiotic sensitivities being performed on the same system. 


\subsection{Statistical methods}

We calculated the sensitivity, specificity, positive predictive value (PPV) and NPV and overall accuracy of our algorithm, using MSU culture as the reference standard (Table 1). The organisms identified are also tabulated (Table 2).

\section{Results}

11 patients $(2 \%)$ were excluded due to the use of indwelling catheters or intermittent self-catheterisation. Of the remaining 460 patients, 161 (35\%) were deemed not to fulfil inclusion criteria as their relapse was not felt to be functionally significant enough to justify corticosteroid treatment. leaving 299 patients for analysis (Figure 2).

The majority of patients were female (4:1), and the average age was 41 years (standard deviation [SD] 10). They were treated with our standard regimen of $1 \mathrm{~g}$ methylprednisolone intravenously for 3 days, or $0.5 \mathrm{~g}$ orally for 5 days. As this was an audit of current practice, ethical approval was not required.

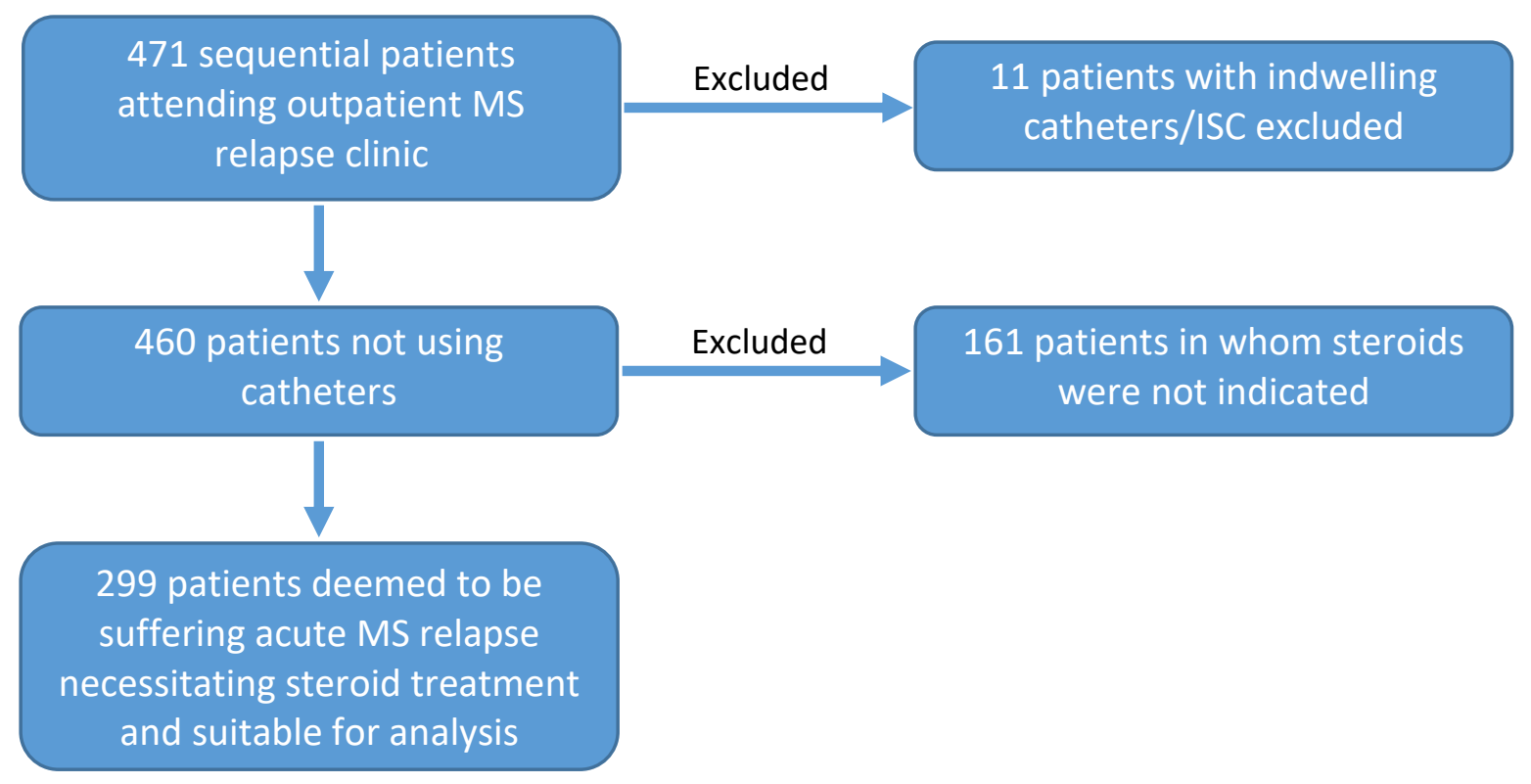

Figure 2. Patients attending outpatient MS relapse clinic who were included in the final analysis.

Performance of urinary dipstick against MSU culture results is shown in Table 1 A-B. 251 patients $(84 \%$, 95\% confidence interval [CI], 79-88) had a negative screening dipstick result which was subsequently confirmed by a negative urine culture. These patients therefore received IV or oral methylprednisolone without antibiotic cover, safely and in a timely manner. 23 patients $(8 \%, 95 \% \mathrm{CI}, 5-11)$ had a positive screening urine result, defined as two or more of raised nitrites or positive LE or cloudy urine. These patients all received oral antibiotics along with steroids, mostly (78\%) nitrofurantoin (100mg for 3-5 days). Following return of culture results, 8 of these patients (3\%, 95\% CI 1-5) were revealed to have significant bacteriuria (Table 1A, true positive). In 7 cases the organism in question was sensitive to the initial antibiotic prescribed. In the remaining case, in which Proteus mirabilis was grown, the patient was contacted within 48 hours to discontinue the nitrofurantoin and change the antibiotic to trimethoprim. 15 patients $(5 \%, 95 \%$ CI, 3-8) had a false positive screening urine. In all cases, antibiotics were discontinued at 48 hours with return of culture results, and none of these patients developed adverse events related to the antibiotics.

\begin{tabular}{|l|l|l|l|}
\hline \multirow{2}{*}{ Urine result } & \multicolumn{2}{|l|}{ MSU } & Total \\
\cline { 2 - 4 } & Positive & Negative & \\
\hline \multicolumn{1}{|l|}{ (A) Per-treatment ( $\geq 2 / 3$ of positive LE, positive nitrites or cloudy urine) } & \multicolumn{2}{l|}{} \\
\hline Positive & 8 & 15 & 276 \\
\hline Negative & 25 & 251 & 299 \\
\hline Total & 33 & 266 & \\
\hline
\end{tabular}




\section{(B) Per-protocol ( $\geq 2 / 3$ of positive LE, positive nitrites or cloudy urine)}

\begin{tabular}{|l|l|l|l|}
\hline Positive & 8 & 13 & 21 \\
\hline Negative & 25 & 253 & 278 \\
\hline Total & 33 & 266 & 299 \\
\hline
\end{tabular}

Table 1. Urinary dipstick and mid-stream urine (MSU) culture results.

A false negative dipstick occurred in 25 patients (8\%, 95\% CI 6-12), who therefore received HDC in the presence of untreated UTI (Table 1A, false negative). On follow-up, however, no cases of infection-related complications (such as pyelonephritis or sepsis) were identified. Appropriate antibiotics were prescribed within 48 hours of steroid administration. Of the 25 false negative tests, 12 were caused by Gram-positive bacteriuria (Table 2).

\begin{tabular}{|l|l|l|l|}
\hline Bacteria & Number of cases & True positives & False negatives \\
\hline Gram negative bacteria & 15 & 6 & 9 \\
\hline E. coli & 2 & 1 & 1 \\
\hline Proteus sp. & 1 & 0 & 1 \\
\hline Enterobacter cloacae & 1 & 0 & 1 \\
\hline Citrobacter koseri & 1 & 0 & 1 \\
\hline Pseudomonas sp. & \multicolumn{5}{|l|}{} \\
\hline Gram positive bacteria & 7 & 1 & 6 \\
\hline Enterococcus sp. & 4 & 0 & 4 \\
\hline Group B streptococcus & 2 & 0 & 2 \\
\hline Staphylococcus haemolyticus & 2 & \\
\hline
\end{tabular}

Table 2. Bacteria identified at mid-stream urine culture $(\mathrm{n}=33)$.

In summary, 33/299 patients ( $11 \%, 95 \%$ CI 8-15) were found to have significant bacteriuria. Table 3A summarises the performance of this refined algorithm: a sensitivity of $24 \%$, with a specificity of $94 \%$; the positive and negative predictive values were $35 \%$ and $91 \%$ respectively. The algorithm's overall accuracy (per-treatment) was $87 \%$.

Two patients were given antibiotics despite not satisfying our screening criteria for UTI. In both patients, urinary dipstick was positive for leucocytes and blood, and it is likely that the criteria were misinterpreted in these cases. Adjusted results are shown in Table 1B. The per-protocol algorithm performance differs minimally (Table 3B).

\begin{tabular}{|l|l|l|l|l|}
\hline & $\begin{array}{l}\text { A) Algorithm v2 } \\
\text { (per-treatment) }\end{array}$ & $\begin{array}{l}\text { B) Algorithm v2 } \\
\text { (per-protocol) }\end{array}$ & $\begin{array}{l}\text { C) Algorithm v1 } \\
\text { (per-treatment) }\end{array}$ & $\begin{array}{l}\text { D) Algorithm v1 } \\
\text { (per-protocol) }\end{array}$ \\
\hline Sensitivity & 24 & 24 & 78 & 80 \\
\hline Specificity & 94 & 95 & 75 & 75 \\
\hline PPV & 35 & 38 & 32 & 32 \\
\hline NPV & 91 & 91 & 96 & 96 \\
\hline Accuracy & 87 & 87 & 78 & 79 \\
\hline Positive likelihood ratio & 4.00 & 4.80 & 3.12 & 3.20 \\
\hline Negative likelihood ratio & 0.81 & 0.80 & 0.29 & 0.27 \\
\hline
\end{tabular}

Table 3. Algorithm performance v2 per-treatment and per-protocol vs. v1 per-treatment and per-protocol, expressed as a percentage.

We went on to do further post-hoc analyses of the per-protocol dataset (as in 2013), to examine the same values for different combinations of: cloudy and/or positive LE and/or positive nitrites, using our per protocol dataset (Table 4A-E). By making the requirement that all three conditions were satisfied (4A), the sensitivity falls further, resulting in $11 \%$ of patients having a delay in their antibiotics. The four combinations 4B-E have low sensitivities (9-15\%), high specificities (92-99\%) and NPV 89-90\%.

\begin{tabular}{|l|l|l|l|l|l|}
\hline & $\begin{array}{l}\text { A) 3/3 of positive } \\
\text { LE, nitrites, cloudy }\end{array}$ & $\begin{array}{l}\text { B) Cloudy and } \\
\text { positive LE }\end{array}$ & $\begin{array}{l}\text { C) Cloudy and } \\
\text { positive nitrites }\end{array}$ & $\begin{array}{l}\text { D) Positive LE } \\
\text { and nitrites }\end{array}$ & E) Cloudy \\
\hline Sensitivity & 6 & 9 & 12 & 15 & 15 \\
\hline Specificity & 99 & 96 & 99 & 99 & 92 \\
\hline PPV & 50 & 21 & 66 & 63 & 19 \\
\hline NPV & 89 & 89 & 90 & 90 & 90 \\
\hline Accuracy & 89 & 86 & 90 & 90 & 83 \\
\hline
\end{tabular}

Table 4. Algorithm performance per-protocol for various combinations of cloudy urine, positive LE and raised nitrites, expressed as percentage. 


\section{Discussion}

UTIs remain a major problem for people with multiple sclerosis (PwMS). They are a common trigger of disease relapses, and are associated with significant patient morbidity through sustained neurological deficits and high rates of hospitalisation[16]. UTIs in MS are also costly, with UK data from 2016/17 showing that non-elective admissions related to UTI resulted in a cost of $£ 930,982[16]$.

For physicians managing an outpatient MS relapse clinic, the potential presence of UTI is problematic for a number of reasons. The desire to provide HDC in a timely manner is understandable given the convincing evidence of shortened duration of deficits in relapses[17]. There is, however, a reluctance to treat patients with a potential UTI with steroids due to fears about exacerbating the infection and leading to systemic sepsis[3,10]. Conversely, co-treating patients indiscriminately with antibiotics is not desirable due to the potential for allergic and other adverse reactions (such as $C$. difficile infection), and the development of antimicrobial resistance.

Our original work on this topic was published over five years ago. While the algorithm $v 1(\geq 1$ of positive nitrites or LE) was effective in allowing us to treat all patients with HDC where clinically indicated, a high false positive rate at $18 \%$ resulted in a significant exposure to unnecessary antibiotics[14]. The issue of multi-drug antimicrobial resistance, well-described in PwMS for over 30 years, has continued to grow since our original paper[18]. Indeed a recent study of patients with neurogenic bladder and asymptomatic bacteriuria or a UTI found that $50 \%$ of strains isolated were multi-drug resistant organisms[19]. Conscious of these issues, we developed an updated algorithm v2 for detection of UTIs in patients with MS relapses. Our objective was to reduce the rate of false positives yielded by the algorithm, while maintaining accuracy and safety.

Our primary comparison is between the per-treatment versions of the two algorithms v1 and v2. As shown in Table 3A and 3C, the overall accuracy of our algorithm increased from $78 \%$ to $87 \%$. There was a large gain in test specificity, though at the expense of a significantly poorer sensitivity ( $78 \%$ dropping to $24 \%$ ). With our overall rate of UTIs remaining consistent across the two studies at $11 \%$, the NPV of algorithm v2 decreased slightly (while remaining $>90 \%$ ) and the positive predictive value (PPV) increased slightly (though remained $<40 \%$ ). Correspondingly, the positive likelihood ratio increased to 4 from 3.12 in v1, while the negative likelihood ratio was also increased at 0.81 (from 0.29 ). In the current cohort of patients, $8 \%$ had a delay in antibiotic provision where it was indicated, compared to $3 \%$ in our previous cohort. Only $4 \%$ of patients received antibiotics unnecessarily, compared to $18 \%$ with our previous algorithm v1. There is little difference in the per-protocol values.

It is worth noting that a large proportion of the false negative cases (12/25 or 48\%) were due to Gram-positive organisms in the MSU culture. It has previously been noted that the LE/nitrite urine dipstick tests are poorly sensitive for Gram-positive organisms[20,21]. This may partly be explained by the fact that most Gram-positive uropathogens, such as Enterococcus faecalis, Staphylococcus saphrophyticus and Group B Streptococcus, do not reduce nitrates. This likely explains the poor sensitivity of our algorithm (just 8\%) for such organisms in the MSU. Recent evidence, however, suggests that the presence of Enterococcus and Group B streptococcus in MSU samples, even at high concentrations, does not correlate with their presence in bladder catheter specimens[22]. These findings are consistent with the belief, held by some practitioners, that these organisms only rarely cause acute cystitis. As such their clinical importance as false negatives in our algorithm must be considered carefully.

How does this paper inform practice? A recent survey of French MS centres revealed that $42 \%$ of centres surveyed delayed HDC treatment in cases of MS relapse where bacteriuria is detected, for fear of triggering systemic sepsis [3]. The importance of timely relapse treatment in terms of reducing patient distress and limiting the duration and severity of relapses cannot be underestimated. As with our previous work, following this simple algorithm for UTI detection allowed all patients to be treated immediately with HDC where clinically indicated.

Although no patient with significant bacteriuria exposed to HDC developed any infectious complications, the safety of HDC use in these patients remains to be established and evidence for adverse outcomes following HDC administration is limited. The new algorithm has improved specificity at the expense of reduced sensitivity, which resulted in 25 patients receiving HDC for up to 48 hours in the presence of significant bacteriuria. It is reassuring, however, that no adverse infectious complications occurred in either our current study or the previous study using the original algorithm[14], despite the increase in number (from 7 to 25) of patients who were not initially treated with antibiotics. Similarly, 15 such patients were treated with HDC in a 2015 observational study, with no increased infective complications[23]. There is limited literature on the risks of high dose corticosteroids 
unmasking untreated UTIs and further research is needed to establish this in the MS population. In the meantime, it is important for clinicians treating patients with HDC to inform patients of potential development of UTI symptoms following therapy, even if urinalysis is initially negative.

This study excluded a small number of patients with catheters (which represented only $2 \%$ of the total number attending the MS relapse clinic in the duration of this study) and as such our algorithm is not validated for use in screening for UTIs in this group of patients. Diagnosis of UTIs in these patients can be challenging. Some conclusions can be drawn from another study auditing 118 MS patients who presented for assessment of a possible relapse. 21 urine dipsticks were abnormal (positive for any of nitrites, leucocytes, blood or protein) and of these, a total of eight patients had catheters. Six cases subsequently had a positive urine culture, whilst only one had no significant growth and another sample was not sent for culture. Whilst this is a small sample, it suggests that a positive urine dipstick is significant for the presence of bacteriuria in patients with catheters[10].

Prevalence of asymptomatic bacteriuria is high in patients with catheters or neurogenic bladder and does not necessarily indicate an infection or routinely require antibiotic treatment. One of the more widely accepted guidelines for UTI diagnosis is from the National Institute on Disability and Rehabilitation Research, mainly directed at patients with spinal cord injuries. These criteria base diagnosis of UTI on presence of significant bacteriuria and at least one of the following symptoms or signs of UTI: pyuria, fever, discomfort or pain over kidney or bladder, urinary incontinence, increased spasticity, cloudy urine with increased odour, malaise, lethargy, and sense of unease. Patients with indwelling or suprapubic catheters may also experience bypassing of urine around the catheter[24]. Other studies in patients with spinal cord injuries have reported absence of pyuria as a strong indicator for no infection[15]. Given the variability in clinical presentation in this group of patients, it is more challenging to apply a single algorithm to screen for UTIs in the context of a potential MS relapse. However, an abnormal urine dipstick and presence of one of the above clinical features may warrant consideration of empirical antibiotic treatment while awaiting formal urine culture result.

\section{Conclusion}

In summary, we believe that our updated algorithm is an improvement, being more accurate overall and resulting in a clear decrease in the prescription of unnecessary antibiotics (from $18 \%$ to $4 \%$ ), whilst retaining patient safety. It allows patients with significant relapses to be treated quickly with HDC in an outpatient setting, while providing a mechanism to reduce unnecessary antibiotic exposure. 


\section{References}

[1] Filippini G, Brusaferri F, Wa S, Citterio A, Ciucci G, Midgard R, et al. Corticosteroids or ACTH for acute exacerbations in multiple sclerosis (Review). Cochrane Database of Systematic Reviews 2009:1-3.

[2] Kisic Tepavcevic D, Pekmezovic T, Dujmovic Basuroski I, Mesaros S, Drulovic J. Bladder dysfunction in multiple sclerosis: a 6-year follow-up study. Acta Neurologica Belgica 2017;117:83-90.

[3] Rouzaud C, Hautecoeur P, Donze C, Heinzlef O, Dinh A, Creange A, et al. Treating asymptomatic bacteriuria before immunosuppressive therapy during multiple sclerosis: Should we do it? Multiple Sclerosis and Related Disorders 2017;18:161-3.

[4] Buljevac D, Flach HZ, Hop WCJ, Hijdra D, Laman JD, Savelkoul HFJ, et al. Prospective study on the relationship between infections and multiple sclerosis exacerbations. Brain: A Journal of Neurology 2002;125:952-60.

[5] Hufschmidt A, Shabarin V, Rauer S, Zimmer T. Neurological symptoms accompanying urinary tract infections. European Neurology 2010;63:180-3.

[6] Correale J, Fiol M, Gilmore W. The risk of relapses in multiple sclerosis during systemic infections. Neurology 2006;67:652-9.

[7] Phé V, Pakzad M, Curtis C, Porter B, Haslam C, Chataway J, et al. Urinary tract infections in multiple sclerosis. Multiple Sclerosis Journal 2016;22:855-61.

[8] Walsh DA, Durance RA. Fatal acute pyelonephritis following pulsed methylprednisolone for rheumatoid arthritis. Annals of the Rheumatic Diseases 1990;49:955-6.

[9] Nikseresht A, Salehi H, Foroughi AA, Nazeri M. Association Between Urinary Symptoms and Urinary Tract Infection in Patients With Multiple Sclerosis. Global Journal of Health Science 2015;8:253.

[10] Mahadeva A, Tanasescu R, Gran B. Urinary tract infections in multiple sclerosis: under-diagnosed and under-treated? A clinical audit at a large University Hospital. American Journal of Clinical Experimental Immunology 2014;3:57-67.

[11] Kupelian AS, Horsley H, Khasriya R, Amussah RT, Badiani R, Courtney AM, et al. Discrediting microscopic pyuria and leucocyte esterase as diagnostic surrogates for infection in patients with lower urinary tract symptoms: Results from a clinical and laboratory evaluation. BJU International 2013;112:231-8.

[12] Lammers RL, Gibson S, Kovacs D, Sears W, Strachan G. Comparison of test characteristics of urine dipstick and urinalysis at various test cutoff points. Annals of Emergency Medicine 2001;38:505-12.

[13] Gorelick MH, Shaw KN. Screening tests for urinary tract infection in children: A meta-analysis. Pediatrics 1999; 104:e54.

[14] Rakusa M, Murphy O, McIntyre L, Porter B, Panicker J, Fowler C, et al. Testing for urinary tract colonization before high-dose corticosteroid treatment in acute multiple sclerosis relapses: prospective algorithm validation. European Journal of Neurology 2013;20:448-52.

[15] Massa LM, Hoffman JM, Cardenas DD. Validity, accuracy, and predictive value of urinary tract infection signs and symptoms in individuals with spinal cord injury on intermittent catheterization. The Journal of Spinal Cord Medicine 2009;32:568-73.

[16] Health IQ Limited. Health episode statistics in multiple sclerosis. 2018.

[17] Lattanzi S, Cagnetti C, Danni M, Provinciali L, Silvestrini M. Oral and intravenous steroids for multiple sclerosis relapse: a systematic review and meta-analysis. Journal of Neurology 2017;264:1697-704.

[18] van Poppel H, Baert L. Treatment of multi-resistant urinary tract infections in patients with multiple sclerosis. Pharmaceutisch Weekblad Scientific Edition 1987;9 Suppl:S76-7.

[19] Togan T, Kurt Azap O, Durukan E, Arslan H. The prevalence, etiologic agents and risk factors for urinary tract infection among spinal cord injury patients. Jundishapur Journal of Microbiology 2014;7:1-8.

[20] Pfaller MA, Koontz FP. Laboratory evaluation of leukocyte esterase and nitrite tests for the detection of bacteriuria. Journal of Clinical Microbiology 1985;21:840-2.

[21] Lewis AL, Kline KA. Gram-Positive Uropathogens, Polymicrobial Urinary Tract Infection, and the Emerging Microbiota of the Urinary Tract. Microbiology Spectrum 2016;4:1-54.

[22] Hooton TM, Roberts PL, Cox ME, Stapleton AE. Voided Midstream Urine Culture and Acute Cystitis in Premenopausal Women. New England Journal of Medicine 2013;369:1883-91.

[23] Donzé C, Dinh A, Heinzlef O, Hautecoeur P. Traiter ou ne pas traiter les bactériuries asymptomatiques avant bolus de méthylprednisolone ? Revue Neurologique 2015;171:669-73.

[24] The prevention and management of urinary tract infections among people with spinal cord injuries. National Institute on Disability and Rehabilitation Research Consensus Statement. January 27-29, 1992., J Am Paraplegia Soc. 15 (1992) 194-204. 